\title{
Recombinant AAV-PR39-mediated hypoxia-inducible factor $1 \alpha$ gene expression attenuates myocardial infarction
}

\author{
LIJUN SUN, YUEWEN HAO, XIAOWEI NIE, JIAN XU, ZHENWU LI, \\ WEI ZHANG, YING LIU and XUEXIN ZHANG
}

Department of Radiology, Xijing Hospital of the Fourth Military Medical University, Xi'an, Shaanxi 710032, P.R. China

Received August 2, 2013; Accepted November 5, 2013

DOI: $10.3892 /$ ijmm.2013.1558

\begin{abstract}
PR39 is an angiogenic masterswitch protein, belonging to the second generation of angiogenic growth factors. However, the role of recombinant adeno-associated virus (AAV) carrying the PR39 fusion gene (AAV-PR39) in acute myocardial infarction remains unclear. Therefore, in this study, we investigated the role of AAV-PR39 in an experimental animal model of acute myocardial infarction. The PR39 gene was fused with the transmembrane peptide, TAT, 6xHis-tag and NT4 signal sequences. AAV-PR39 was then obtained by calcium phosphate co-precipitation. A total of 18 healthy Chinese mini pigs were randomly divided into an experimental groups (the AAV-PR39-treated group) and a control group [phosphated-buffered saline (PBS)-treated group]. Following the induction of myocardial infarction, enhanced 3.0T MR imaging was performed to observe the changes in myocardial signal intensity at $0 \mathrm{~h}, 1,2$ and 3 weeks. The expression of hypoxia-inducible factor- $1 \alpha(\mathrm{HIF}-1 \alpha)$ in the myocardial tissues was determined by SABC immunohistochemistry. In addition, in vitro experiments using CRL-1730 endothelial cells transfected with AAV vector containing NT4-TAT-His-PR39 revealed that the AAV-PR39-treated group had a significantly higher expression of HIF-1 $\alpha$ compared with the control group. Moreover, PR39 regulated the HIF- $1 \alpha$-induced expression of angiogenic growth factors. Under hypoxic conditions, the antiapoptotic effects in the AAV-PR39 group were more pronounced than those observed in the control (PBS-treated) group. In vivo, the enforced expression of recombinant PR39 elevated the level of HIF-1 $\alpha$ under hypoxic conditions and decreased the size of the infarcted areas by upregulating the expression of HIF-1 $\alpha$ in the areas surrounding the infarct area. Taken together, our data demonstrate that the recombinant AAV-PR39-mediated HIF-1 $\alpha$ expression attenuates myocardial infarction, indicating
\end{abstract}

Correspondence to: Dr Lijun Sun, Department of Radiology, Xijing Hospital of the Fourth Military University, No. 15 Changle West Road, Xi'an, Shaanxi 710032, P.R. China

E-mail: lijunsunxian@126.com

Key words: PR39, gene therapy, hypoxia-inducible factor $1 \alpha$, myocardial infarction that AAV-PR39 may serve as a novel therapeutic agent for the treatment of myocardial infarction.

\section{Introduction}

Acute myocardial infarction is a serious threat to human health. With the changes in dietary structure, the incidence of and mortality rates due to myocardial infarction have shown an increasing trend over the years (1). The utilization of genetic engineering technology to facilitate angiogenesis in the infarcted area, to rescue the dying myocardium and to improve cardiac function has become a focus of research in recent years (2). A large number of experimental and clinical studies have demonstrated that genetic engineering technology can be used to improve local blood infusion and restore impaired heart function in the ischemic myocardium (3-5). However, previous studies have usually focused on the role of a single factor, such as vascular endothelial growth factor (VEGF) or basic fibroblast growth factor (bFGF) in angiogenesis. The long-term and high expression of VEGF has been shown to result in the continuous increase in the levels of VEGF in the blood, which may cause side-effects, such as hemangioma, retinal disease and various types of cancer. These side-effects (disadvantages) are the obstacles of gene therapy (6). Muinck et al suggested that the second generation of vascularderived factor and therapeutic gene transfer may enhance the expression of multiple angiogenic factors. PR39 is an angiogenic masterswitch protein (7), belonging to the second generation of angiogenic growth factors. Li et al demonstrated that exogenous PR39 can selectively inhibit the ubiquitin/proteasome-dependent degradation of hypoxia-inducible factor (HIF)-1 $\alpha$ and can increase the intracellular expression of VEGF, kinase insert domain receptor (KDR) and fibroblast growth factor receptor 1 (FGFR1) (8).

In the present study, we successfully enforced the expression of the PR39 fusion gene into CRL-1730 endothelial cells. Subsequently, we investigated the effects of PR39 using cultured CRL-1730 cells and an animal (pig) model of myocardial infarction. Our results revealed that the adeno-associated virus (AAV)-mediated expression of PR39 enhanced the expression of HIF-1 $\alpha$ and inhibited apoptosis under hypoxic conditions in the CRL-1730 cells. PR39 regulated the HIF-1 $\alpha$ induced expression of angiogenic growth factors. Moreover, compared with the control group, the infarcted areas of the pigs treated with AAV-PR39 were significantly decreased and 
HIF-1 $\alpha$ expression was markedly elevated, thus indicating that treatment with AAV-PR39 exerts protective effects against myocardial infarction.

\section{Materials and methods}

Strains, plasmids and cloning vectors. E. coli TOP10 cells, T/TAT-His vector, pBV220/NT4 vector, AAV plasmid (pSSHG-CMV), helper virus pAAV/Ad and packaging plasmid PFG140 were purchased from Xi'an Huaguang Bioengineering Co. (Xi'an, China). T4 DNA ligase, restriction enzymes (NaeI, BamHI, EcoRI and Eco721), Taq DNA polymerase, and the pGEM-T-Easy vector were purchased from Amersham Life Sciences (Pittsburgh, PA, USA).

Cell culture and hypoxia. The CRL-1730 vascular endothelial cells were purchased from the Cell Bank of Shanghai Institutes for Biological Sciences, Chinese Academy of Sciences, Shanghai, China. The cells were cultured in RPMI-1640 medium (Invitrogen, Carlsbad, CA, USA) supplemented with $10 \%$ FBS. Experiments were performed on cells at 5 to 6 passages. After the cells had grown to confluence, they were placed in a quiescent medium for $24 \mathrm{~h}$. Hypoxia was induced with the use of a modular incubator chamber provided by the Department of Pathology at the Fourth Military Medical University, Xi'an, China. The oxygen level in the chamber was monitored with an oxygen analyzer and remained at $1-3 \%$ for up to $72 \mathrm{~h}$.

Construction of expression box containing NT4-TATHis-PR39. The transmembrane peptide, TAT, was fused with the C-terminus of the NT4 signal peptide and 6xHis-tag was directly fused at the C-terminus of TAT. PR39 was connected downstream of NT4-TAT-His, resulting in the expression box, NT4-TAT-His-PR39. The R39 sequences were amplified using the following primers: forward 1, 3'-CCA CGT GAG GAG ACG TCC CCG ACC CCC ATA TTT GCC-5' and forward 2, 5'-CCA TAT TTG CCA AGG CCA AGG CCA CCT CCG TTC TTC CCA CCA AGG CTC CCA CC-3'; reverse 1, 3'-CCG TGG TGG GAA CCT TGG TGG GAA CCC TGG TGG AAT GCG TGG TGG GAG CC-5' and reverse 2, 5'-CGG ATC CTC AGG GGA ACC GTG GTG GGA ACC-3'. The middle region of PR39 was amplified by primers $\mathrm{F} 2$ and R1, the 5-terminus of PR39 was amplified by primer F1 and the 3-terminus of PR39 was amplified by primer R2. Following two steps of PCR, complete sequences of PR39 were obtained. PCR included 30 cycles of $94^{\circ} \mathrm{C}$ for $60 \mathrm{sec}, 40^{\circ} \mathrm{C}$ for $60 \mathrm{sec}$ and $72^{\circ} \mathrm{C}$ for $90 \mathrm{sec}$ followed by $10 \mathrm{~min}$ of extension. The PCR product was ligated into the pGEM-T Easy vector, resulting in pGEM-T/PR39, which was subsequently transfected into competent $E$. coli TOP10 cells.

Construction of pBV220/NT4-TAT-His-PR39 vector. The pGEM-T/PR39 vector was double digested with Eco721 and BamHI. The PR39 fragment was excised and ligated into the T/TAT-His vector digested with identical restriction enzymes. The ligation products were transformed into the TOP10 strain and positive clones containing T/TAT-His-PR39 were obtained. T/TAT-His-PR39 was double digested with $\mathrm{NaeI}$ and BamHI and the excised product was ligated into pBV220/NT4, thus creating the vector, pBV220/NT4-TAT-His-PR39. The successful construction of the vector was confirmed by DNA sequencing.

Construction of AAV vector containing NT4-TAT-His-PR39. Both pSSCMV and pBV220/NT4-TAT-His-PR39 were double digested with EcoRI and BamHI and the excised NT4-TATHis-PR39 fragment was ligated into linear pSSCMV that is the AAV promoter, thus creating the vector, pSSCMV/ NT4-TAT-His-PR39. HEK-293 cells were co-transfected with pSSCMV/NT4-TAT-His-PR39, packaging plasmid PFG10 and helper virus plasmids pAAV/Ad for $72 \mathrm{~h}$. The transfected cells were harvested, and three turns of repeated freezing and thawing were performed. The cells were subsequently lysed by sonication. Following centrifugation at 5,000 rpm for $15 \mathrm{~min}$, cell debris was removed and the derived virus suspension was inactivated by heating at $56^{\circ} \mathrm{C}$ for $30 \mathrm{~min}$. Purified recombinant AAV was then harvested.

Quantitative reverse transcription PCR ( $q R T-P C R)$. Total RNA was extracted using the RNA Plus kit (Takara, Dalian, China). Total RNA $(1 \mu \mathrm{g})$ was used for cDNA synthesis with a QuantiTech Reverse Transcription kit (Takara) according to the manufacturer's instructions. The PCR mixture contained cDNA, generated from $2 \mathrm{ng}$ of total RNA, $0.1 \mathrm{nmol} / 1$ forward and reverse primer mix and SYBR-Green I reagent. The specific primers used were as follows: HIF-1 $\alpha$ sense, 5'-AGC CAG ACG ATC ATG CAG CTA CTA-3' and antisense, 5'-GAG TAC TTG CGC TCA GGA GGA -3'; angiopoietin-2 sense, 5'-GGG CAT AAT TGT GCT TGA CTG G-3' and antisense, 5'-ATG GTC TTT AGA ATT GGG TCA CTG G-3'; endostatin sense, 5'-CTC AAT GCA GAG CAC GAT GT-3' and antisense, 5'-TGT TCT CAG GCT CTG AGG GT-3'; thrombospondin-2 sense, 5'-TGG AAG GAC TAC ACG GCC TAT AG-3' and antisense, 5'-TAG GTT TGG TCA TAG ATA GGT CCT GAG T-3'; and $\beta$-actin sense, 5'-ATT GCC GAC AGG ATG CAG A-3' and antisense, 5'-GAG TAC TTG CGC TCA GGA GGA-3'. Quantitative PRC assays were performed using the Bio-Rad iQ5 Quantitative PCR system (Bio-Rad, Hercules, MA, USA) and SYBR Premix Ex Taq kit (Takara). Amplification was carried out for one cycle of $2 \mathrm{~min}$ at $50^{\circ} \mathrm{C}$ and one cycle of $2 \mathrm{~min}$ at $94^{\circ} \mathrm{C}$ followed by 40 cycles of a 2-step loop: $15 \mathrm{sec}$ at $95^{\circ} \mathrm{C}$ and $30 \mathrm{sec}$ at $60^{\circ} \mathrm{C}$. Relative quantification of the PCR products was calculated following normalization to $\beta$-actin.

Western blot analysis. Total protein extracts were prepared using RIPA lysis buffer (Beyotime, Nantong, China) according to the manufacturer's instructions. The protein concentration in the lysates was evaluated using a BCA protein assay kit (Beyotime). For western blot analysis, the proteins lysates (30 $\mu \mathrm{g} / \mathrm{lane}$ ) were separated by SDS-PAGE and transferred onto polyvinylidene difluoride membranes (Whatman Schleicher \& Schuell, Middlesex, UK). The membranes were blocked for $20 \mathrm{~min}$ at room temperature with TBS blocking buffer. The blots were then incubated overnight at $4^{\circ} \mathrm{C}$ with each primary antibody (anti-PR39, antiHIF-1 $\alpha$, anti-angiopoietin-2, antiendostatin and anti-thrombospondin-2 antibody) followed by incubation for $1 \mathrm{~h}$ with horseradish peroxidase-conjugated secondary antibody. After washing, the sites of antibody binding were visualized with chemiluminescence reagent 
(Boehringer Mannheim, Mannheim, Germany) and the relative levels of each protein to $\beta$-actin were calculated.

siRNA transfection. For the targeted knockdown of HIF-1 $\alpha$, siRNA-HIF-1 $\alpha$ was designed and synthesized by Genetimes Technology Inc. (Shanghai, China). The nucleotide sequences were as follows: siRNA-HIF-1 $\alpha$, 5'-TCG AGG AAG GAA CCT GAT GCT TTA TTC AAG AGA TAA AGC ATC AGG TTC CTT CTT A-3' (sense) and 5'-CTA GTA AGA AGG AAC CTG ATG CTT TAT CTC TTG AAT AAA GCA TCA GGT TCC TTC C-3' (antisense). As a control for siRNA-HIF-1 $\alpha$, a corresponding random siRNA sequence was used (positive strand, 5'-TCG AGG GAG ACC GGA TTT GAT CTA TTC AAG AGA TAG ATC AAA TCC GGT CTC CTT A-3' and negative strand, 5'-CTA GTA AGG AGA CCG GAT TTG ATC TAT CTC TTG AAT AGA TCA AAT CCG GTC TCC C-3'). For transfection, the CRL-1730 cells were seeded in each cell of a 24-well microplate, grown for $24 \mathrm{~h}$ till they reached $50 \%$ confluence, and incubated with a mixture of 6 pmol siRNAHIF- $1 \alpha$ and $1 \mu$ l Lipofectamine RNAi-MAX (Invitrogen, Carlsbad, CA) in $100 \mu \mathrm{l}$ serum-free medium 254 at $37^{\circ} \mathrm{C}$ with $5 \% \mathrm{CO}_{2}$. Twenty-four hours later, the transfection efficiency was examined by measuring the levels of HIF-1 $\alpha$ by qRT-PCR and western blot analysis, as described above.

Immunohistochemistry. CRL-1730 cell suspension was transferred into the culture dishes with coverslips and RPMI-1640 medium containing $10 \%$ FBS was added. Following $24 \mathrm{~h}$ of culture, $100 \mu 1$ of AAV-NT4-TAT-His-PR39 $\left(3.4 \times 10^{9} \mathrm{pfu}\right)$ or control vector were used to infect the CRL-1730 cells for $60 \mathrm{~h}$. The coverslips with the cells were rinsed with PBS twice and fixed with acetone for $15 \mathrm{~min}$. After three rinses with PBS, the coverslips were cultured in an incubator containing $0.75 \% \mathrm{H}_{2} \mathrm{O}_{2}$ for $30 \mathrm{~min}$, and then blocked with $20 \%$ normal bovine serum in incubation buffer $(0.5 \%$ Triton X-100 in $0.1 \mathrm{M}$ PBS). The coverslips were then supplemented with primary monoclonal anti-PR39 or anti-HIF-1 $\alpha$ (1:500 dilution) antibody and incubated at $4^{\circ} \mathrm{C}$ overnight. After extensive washes, secondary goat anti-rabbit IgG was added. A diaminobenzidine (DAB) staining kit was used to detect a positive reaction by producing a brown color. The protein expression level was quantified by the optical density (OD) of brown color calculated using Image Pro Plus (IPP) software.

Detection of apoptosis by flow cytometry. The CRL-1730 cells were washed twice with cold PBS and resuspended at a concentration of $1 \times 10^{6} / \mathrm{ml}$. The cells $(100 \mu \mathrm{l})$ were supplemented with $5 \mu \mathrm{l}$ Annexin V-FITC and $5 \mu \mathrm{l}$ propidium iodide (PI). After gentle mixing, the cells were incubated in the dark for $15 \mathrm{~min}$. Staining buffer $(400 \mu \mathrm{l})$ was then added to the cells and apoptosis was analyzed immediately by flow cytometry.

Development of animal (pig) model of myocardial infarction. The animal experiments caried out in this study conformed to the guidelines for Laboratory Animals issued by the Fourth Military Medical University. The present study was performed with the approval of the Animal Ethics Committee of the Fourth Military Medical University (Certification no. 0145). All surgerical procedures were performed under sodium pentobarbital anesthesia, and all efforts were made to minimize the suffering of the animals. A total of 18 Chinese mini pigs (weighing 20-25 kg, provided by the Fourth Military Medical University Experimental Animal Center) were randomly divided into an experimental group (AAV-PR39-treated group, $\mathrm{n}=9$ ) and a control group [normal saline (PBS)-treated group, $\mathrm{n}=9$ ]. After $12 \mathrm{~h}$ of pre-operative fasting and $4 \mathrm{~h}$ of water deprivation, $2 \mathrm{ml}$ of sumianxin II (Animal Husbandry Industry and Animal Health Products Co., Ltd., Jilin, China) and $18 \mathrm{ml}$ of $5 \%$ pentobarbital sodium $(1 \mathrm{ml} / \mathrm{kg}$ ) (Fourth Military Medical University) were injected intramuscularly. After successful anesthesia, the pigs were fixed in a supine position on a homemade wooden stand and placed on a DSA catheter bed (Axiom Artis dBA; Siemens, Erlangen, Germany). Intravenous access was established and atropine $(1 \mathrm{mg})$ was injected intramuscularly. The the pig model of myocardial infarction was then constructed as previously described (6). One milliliter of AAV/HRE-PR39 containing $3 \times 10^{9}$ pfu (experimental group) or $1 \mathrm{ml}$ of PBS (control group) was injected into the left anterior descending artery of each mini pig via a $3 \mathrm{~F}$ microcatheter. ECG monitoring was performed during the surgery. MRI examination was performed for the left ventricles after $0,1,2$ and 3 weeks of the bandaging of the right thigh or ligation of the right femoral artery. All the pigs were sacrificed 3 weeks after surgery and the normal as well as diseased cardiac tissues were obtained.

Cardiac MRI examination. A superconductive scanner (Magnetom Trio 3.0T MRI, Siemens, AG, Germany) and body coil were used for the MRI examination. The short and long axis of the heart (view of two-chamber, four-chamber) was determined by conventional MRI scanning. Subsequently, HASTE sequential scanning was performed for axial and coronal views. The following scanning parameters were used: repetition time (TR)/echo time (TE), 649/52 msec; thickness, $6 \mathrm{~mm}$; no spacing; field of view (FOV), $380 \mathrm{~mm}$; rectangular field of view (RFOV), 68.8\%; acquisition window, 673 msec; trigger pulse, 2; trigger delay, $24 \mathrm{msec}$; flip, $160^{\circ}$. Left ventricular short axis view and long axis view was examined by magnetic resonance sensitive sequence (T2-weighted TrueFISP Imaging of Myocardium) to understand the myocardial edema. The following scanning parameters were used: TR/TE, 281.95/1.09 msec; thickness, $6 \mathrm{~mm}$; no spacing; Matrix, 256x256; FOV, $360 \mathrm{~mm}$; RFOV, 75\%; acquisition window, 715 msec; trigger pulse, 2; trigger delay, $433 \mathrm{msec}$; flip, $160^{\circ}$. Myocardial delayed perfusion imaging was performed by $2 \mathrm{D}$ TrueFISP-PSIR sequences. Contrast agents were injected at a speed of $1 \mathrm{ml} / \mathrm{sec}$ and 10 min after injection, scanning commenced. The following scanning parameters were used: TR/TE, 382.24/1.09 msec; thickness, $6 \mathrm{~mm}$; no spacing; matrix, 256x256; FOV, $360 \mathrm{~mm}$; RFOV, 75\%; acquisition window, $750 \mathrm{msec}$; trigger pulse, 2; trigger delay, $300 \mathrm{msec}$; flip, $60^{\circ}$.

Statistical analysis. Data were analyzed using SPSS 13.0 software. The data of each group were tested for normal distributions first. Subsequently, the Student's t-test was carried out for paired samples. The results are presented as the means \pm SD. All reported P-values were two tailed, and $\mathrm{P}<0.05$ was considered to indicate a statistically significant difference. 

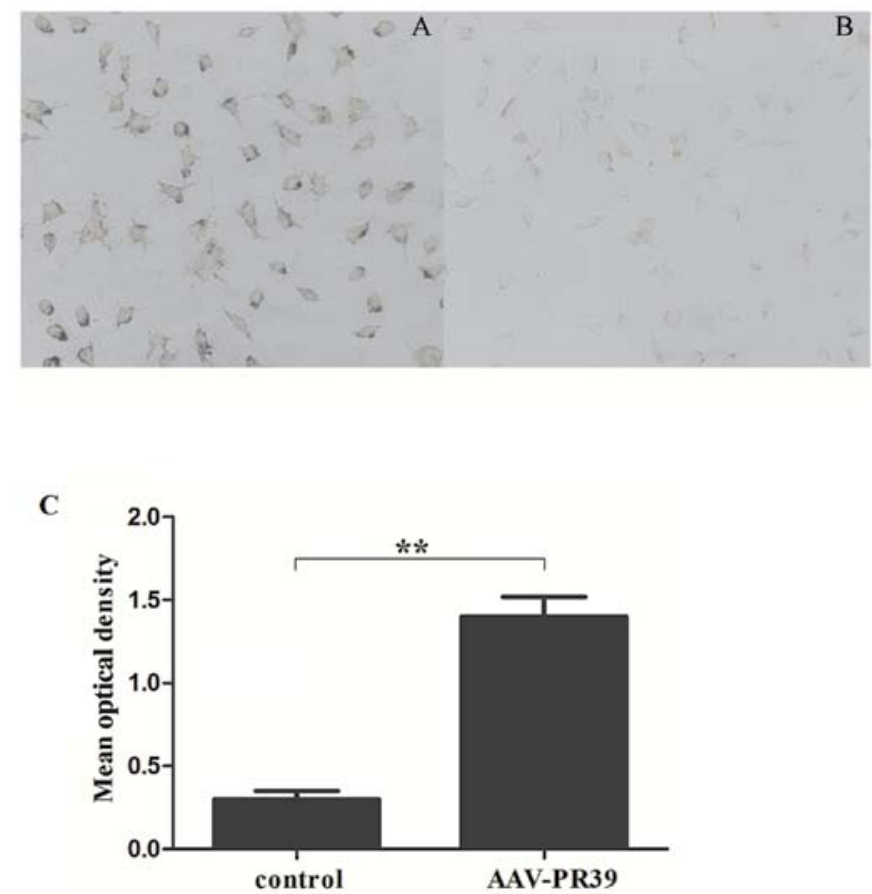

Figure 1. Expression of PR39 in CRL-1730 cells as detected by immunocytochemistry. PR39 was detected in the cytoplasm of the cells infected with AAV-PR39 (A), but was not detected in the control cells (B). (C) The mean optical density (OD) value of PR39 in the AAV-PR39-treated group was markedly higher than that in the control (PBS-treated) group. ${ }^{* *} \mathrm{P}<0.01$ compared with the control group.

\section{Results}

Expression of exogenous PR39 in endothelial cells. The NT4-TAT-His-PR39 fragment was ligated into the linear pSSCMV that is the AAV promoter, which triggered the expression of PR39 in the CRL-1730 cells. As shown by immunohistochemistry, a large amount of brown particles were observed in the AAV-PR39-treated group, while only a few brown particles were observed in the control group (Fig. 1A and B). Western blot analysis revealed that the protein level of PR39 was markedly increased in the AAV-PR39-treated group (Fig. 2C). Moreover, quantitative analysis indicated that the OD value of PR39 was markedly elevated in the AAV-PR39-treated group, as compared with the control group $(\mathrm{P}<0.001)$ (Fig. 1C). These results indicated that the AAV vector containing NT4-TAT-His-PR39 was successfully transfected into the CRL-1730 cells.

Effect of PR39 on the expression of HIF-1a under hypoxicconditions. To determine whether PR39 induces HIF-1 $\alpha$ expression, we determined the HIF- $1 \alpha$ expression levels in CRL-1730 cells. Under hypoxic conditions, the HIF-1 $\alpha$ protein was observed and was mainly expressed in the nucleus. Brown staining of the CRL-1730 cells in the AAV-PR39-treated group was stronger than that in the control group (Fig. 2A and B). Western blot analysis indicated that the level of HIF-1 $\alpha$ expression was markedly elevated in the AAV-PR39-treated group (Fig. 2C). Compared with the control (PBS-treated) group, the OD value for HIF-1 $\alpha$ protein expression in the AAV-PR39treated group was markedly elevated $(\mathrm{P}<0.05)($ Fig. 2D). These
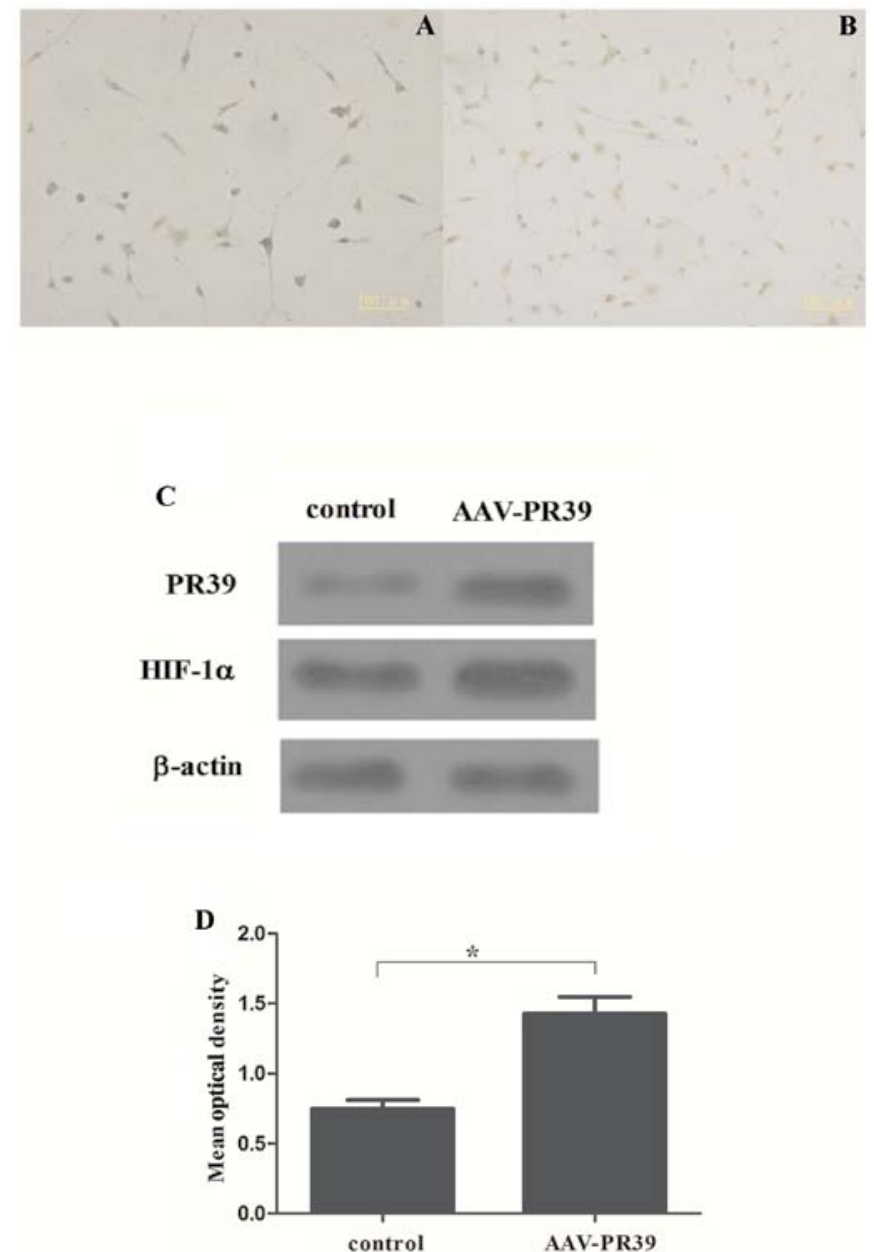

Figure 2. Expression of hypoxia-inducible factor (HIF)-1 $\alpha$ in CRL-1730 cells under hypoxic conditions. Immunocytochemical detection of HIF-1 $\alpha$ in the CRL-1730 cells infected with (A) AAV-PR39 and (B) the control cells. (C) The level of HIF-1 $\alpha$ expression were dramatically elevated in the AAVPR39-treated group. (D) The mean optical density (OD) value of HIF-1 $\alpha$ in the AAV-PR39-treated group was significantly higher than that in the control (PBS-treated) group. ${ }^{*} \mathrm{P}<0.05$ compared with the control group.

results demonstrated that PR39 enhanced the expression of HIF-1 $\alpha$ in the CRL-1730 cells.

PR39 regulates the HIF-1 $\alpha$-induced expression of angiogenic growth factors. We found that PR39 enhanced the expression of HIF-1 $\alpha$ in CRL-1730 cells. Hypoxia regulates the expression of various genes participating in the various steps of angiogenesis (9). Therefore, in this study, the levels of angiopoietin-2, endostatin and thrombospondin-2 were analyzed to determine the effects of HIF-1 $\alpha$ on the expression of angiogenic growth factors. qRT-PCR analysis revealed that the HIF-1 $\alpha$ and angiopoietin-2 mRNA levels were markedly decreased by siRNA-HIF-1 $\alpha$, whereas the endostatin and thrombospondin-2 mRNA levels were markedly increased by siRNA-HIF-1 $\alpha$ $(\mathrm{P}<0.05)$ (Fig. 3A). Consistent with the results from qRT-PCR, the downregulation of HIF-1 $\alpha$ and angiopoietin-2 was also observed following treatment of the CRL-1730 cells with siRNAHIF-1 $\alpha$, whereas endostatin and thrombospondin-2 levels were markedly increased by siRNA-HIF-1 $\alpha$ (Fig. 3B). In addition, siRNA-HIF-1 $\alpha$ combined with PR39 upregulated the expression 


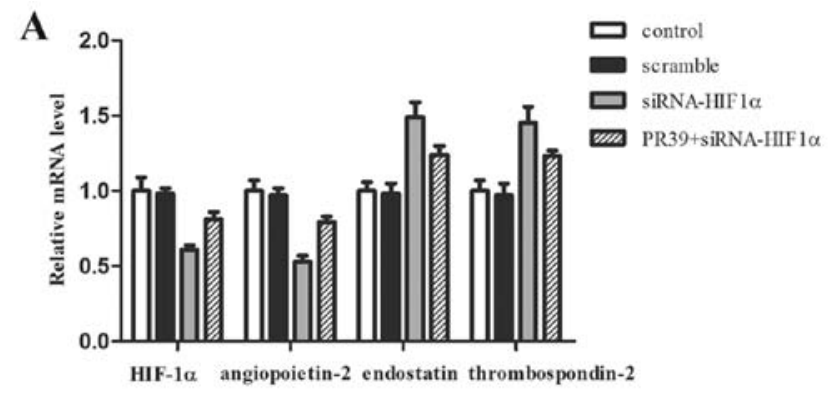

B

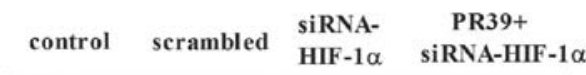

HIF-1 $\alpha$

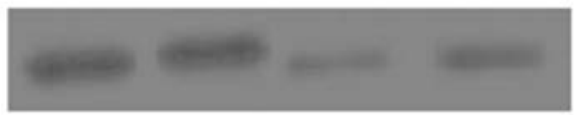

angiopoietin-2

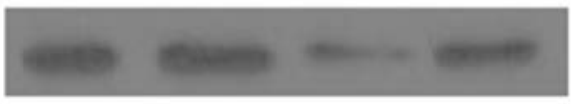

endostatin

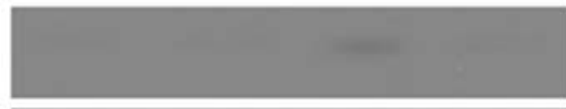

thrombospondin-2

$\beta$-actin

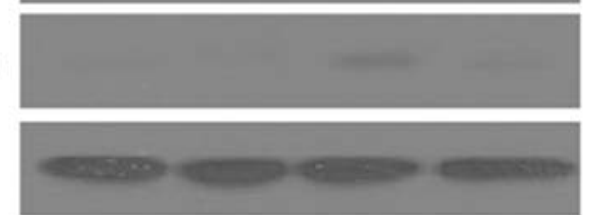

Figure 3. PR39 regulates the hypoxia-inducible factor-1 $\alpha$ (HIF-1 $\alpha)$-induced expression of angiogenic growth factors. (A) Relative mRNA level of angiogenic growth factors in cells treated with siRNA-HIF-1 $\alpha$ and PR39 combined with siRNA-HIF-1 $\alpha$; (B) represent western blots of angiogenic growth factors. The expression levels of proteins were normalized based on the $\beta$-actin levels. ${ }^{*} \mathrm{P}<0.05$ compared with the control.

of HIF- $1 \alpha$ and angiopoietin-2 and downregulated the expression of endostatin and thrombospondin-2 ( $\left.{ }^{*} \mathrm{P}<0.05\right)$, as compared with the siRNA-HIF-1 $\alpha$-treated group (Fig. 3A and B). Taken together, these results demonstrated that PR39 regulated the HIF-1 $\alpha$-induced expression of angiogenic growth factors.

PR39 inhibits hypoxia-induced apoptosis. A previous study reported that PR39 inhibits hypoxia-induced apoptosis in bovine aortic endothelial cells (19). Thus, in this study, we examined the effects of AAV-PR39 on hypoxia-induced apoptosis in CRL-1730 cells. Flow cytometry revealed that the number of apoptotic cells markedly decreased in the AAV-PR39-treated CRL-1730 cells, as compared with the control group (Fig. 4A and B). Quantitative analysis demonstrated that the percentage of apoptotic cells after $72 \mathrm{~h}$ of hypoxia was $32.58 \pm 0.39 \%$ in the untreated cells and $7.35 \pm 0.43 \%$ in the cells pre-treated with AAV-PR39 $(\mathrm{P}<0.01)$ (Fig. 4C). These results indicated that PR39 inhibited apoptosis under hypoxic conditions in the CRL-1730 cells.

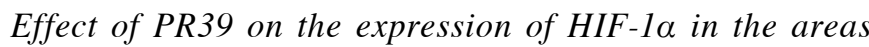
surrounding areas the infarcted area. We investigated whether PR39 affects the infarct area in vivo using a pig model of myocardial infarction. Three weeks after the induction of myocardial infarction, we measured the infarct areas and the results revealed that the size of the infarct areas $\left(167.4 \pm 5.42 \mathrm{~mm}^{2}\right)$ was significantly smaller in the AAV-PR39- treated group, as compared with the control (PBS-treated) group $\left(325.17 \pm 6.42 \mathrm{~mm}^{2}\right)(\mathrm{P}<0.01)$. Furthermore, the size of the infarct area was not significantly altered at the different time points $\left(0 \mathrm{~h}, 342.6 \pm 6.81 \mathrm{~mm}^{2}\right.$; 1 week, $337.4 \pm 6.22 \mathrm{~mm}^{2} ; 3$ weeks, $325.17 \pm 6.42 \mathrm{~mm}^{2}, \mathrm{P}>0.05$ ) in the control (PBS-treated) group. By contrast, in the AAV-PR39-treated group, the size of the infarct area was markedly reduced as time progressed after the induction of myocardial infarction $\left(0 \mathrm{~h}, 326.25 \pm 5.92 \mathrm{~mm}^{2}\right.$; 1 week, $273.6 \pm 4.86 \mathrm{~mm}^{2} ; 3$ weeks, $167.4 \pm 5.42 \mathrm{~mm}^{2}, \mathrm{P}<0.01$ ) (Fig. 5). To investigate the mechanisms involved, we detected the expression of HIF-1 $\alpha$. At 21 days after the induction of myocardial infarction, HIF-1 $\alpha$ expression was detected by immunohistochemistry, as illustrated in Fig. 6A and B. Brown color indicates HIF-1 $\alpha$ protein staining and blue color indicates myocardial nuclear staining in the diseased cardiac tissues. We then examined the expression of HIF-1 $\alpha$ by western blot analysis; the upregulation of HIF-1 $\alpha$ in cardiac tissues was also demonstrated by western blot analysis (Fig. 6C). Quantitative analysis using IPP software indicated that the OD value for HIF-1 $\alpha$ expression in the AAV-PR390-treated group was significantly higher than that in the control group $(\mathrm{P}<0.01)$ (Fig. 6D). These results suggested that PR39 decreased the size of the infarct area by upregulating the expression of HIF-1 $\alpha$ in the areas surrounding the infarct area.

\section{Discussion}

PR39, a proline (P)- and arginine (R)-rich peptide implicated in wound healing and in the protection against myocardial ischemia, was originally isolated from the pig intestine and identified in neutrophil azurophilic granules and macropages $(10,11)$. During the early stages of myocardial infarction, PR39 can reduce the infarct size (12), promote angiogenesis in ischemic tissue, establish an effective collateral circulation and prevent microcirculation dysfunction (13). Currently, studies on the expression of biological short peptides are limited. Thus, in this study, we fused the NT4 signal peptide upstream of PR39. The NT4 signal peptide can recognize the intracellular activation center (14), thus achieving the eukaryotic expression of PR39. To date, the AAV vector is frequently used to investigate the biological effects of the PR39 peptide. The advantage of AAV lies in its non-toxicity, non-immunogenicity, non-pathogenicity and infectivity of non-dividing cells. In addition, the long-term and stable expression of the genes carried by AAV can be achieved in host cells $(15,16)$. Therefore, in this study, we utilized AAV as a vector to carry and deliver the PR39 gene into the host cells.

Carmeliet et al demonstrated that HIF-1 $\alpha$ is highly expressed under hypoxic conditions. In addition, hypoxic conditions can induce an upregulation in the expression of a number of angiogenesis-related genes, including VEGF, VEGF receptor [Fms-related tyrosine kinase 1 (FLT1) and KDR] and FGFR1 $(17,18)$. In this study, we demonstrated that HIF-1 $\alpha$ was maintained at a relatively high level in endothelial cells under hypoxic conditions. In addition, HIF-1 $\alpha$ expression in the AAV-PR39-treated group was higher than that in the control (PBS-treated) group, indicating that PR39 enhanced the expression of HIF-1 $\alpha$ in the cells under hypoxic conditions. One of the possible mechanisms involved may be that PR39 selectively inhibits the ubiquitin/proteasome- 

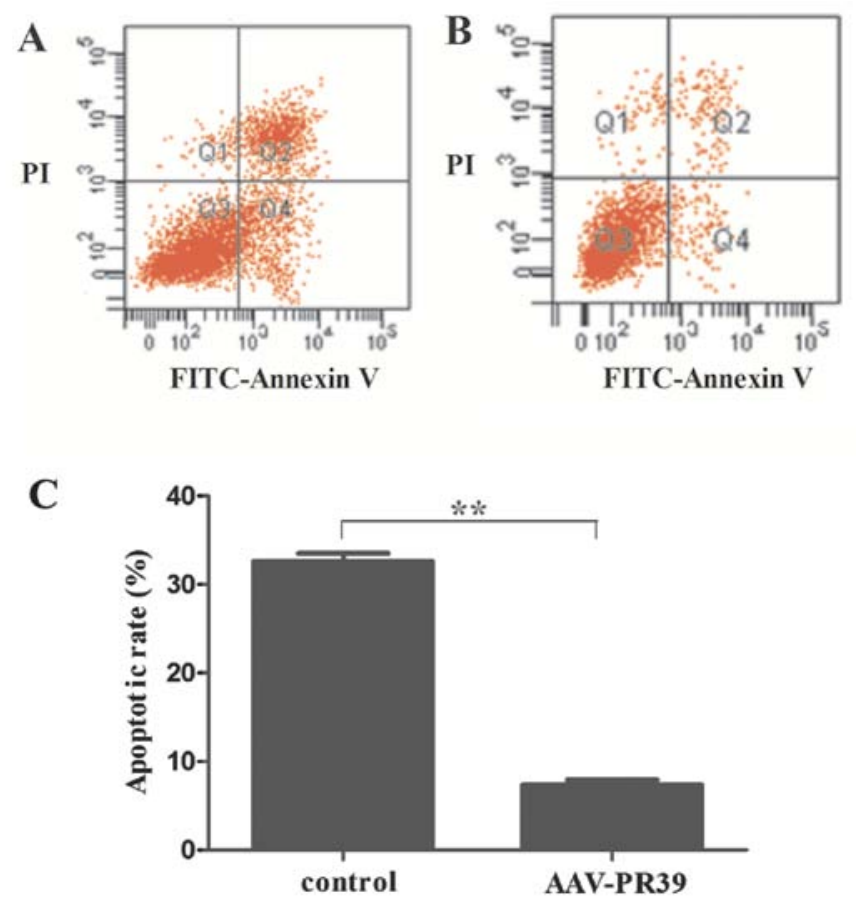

Figure 4. Apoptosis of CRL-1730 cells in the experimental and control groups. Flow cytometric analysis of apoptosis in (A) the control cells (treated with PBS) and (B) cells infected with AAV-PR39. (C) Statistical analysis revealed that apoptotic rate in the AAV-PR39-treated cells was significantly lower than that in the control group. ${ }^{* *} \mathrm{P}<0.01$ compared with the scontrol group.

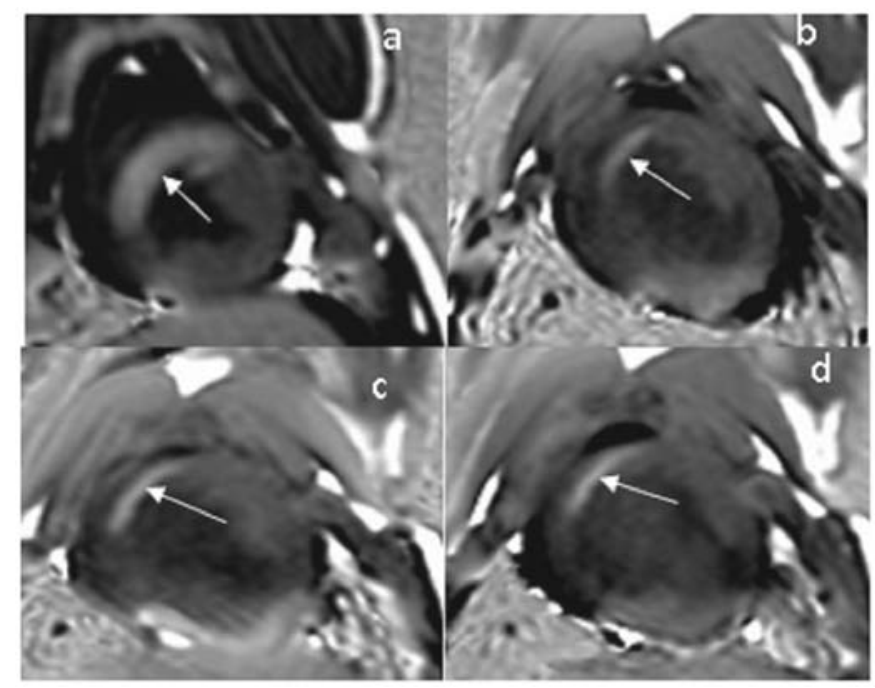

Figure 5. Changes in the size of the infarct area in ( $a$ and $b$ ) the experimental and (c and d) the control groups. (a and c) Changes in the left ventricular infarct size at $0 \mathrm{~h}$; (b and d) changes in the left ventricular infarct size at 3 weeks. In the experimental group, the size of the infarct area was significantly reduced at 3 weeks after the induction of acute myocardial infarction, whereas in the control group, the infarct size was not altered significantly.

dependent degradation of HIF-1 $\alpha$ and enhances the expression of VEGF, KDR, FLT-1 and FGFR1 (8), thereby initiating the expression of a series of growth factors.

Hypoxia is the cellular stress which occurs when oxygen demand exceeds supply. As a homeostatic response to this challenge, several classes of genes are upregulated, which encode proteins involved in angiogenesis, such as VEGF
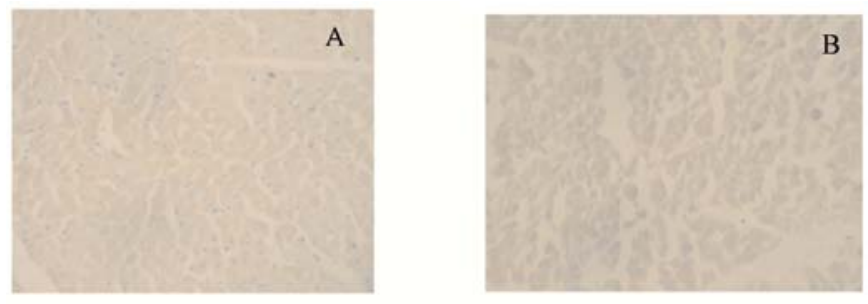

C

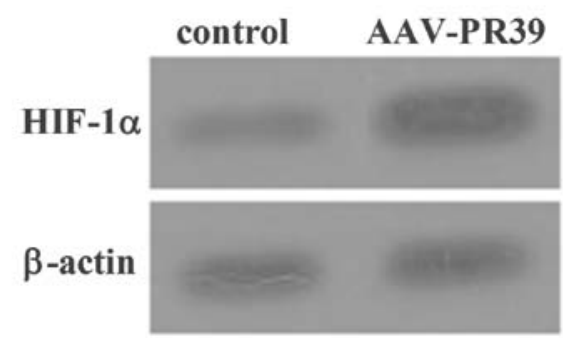

D

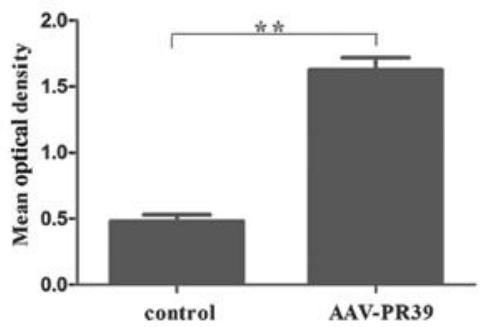

Figure 6. Hypoxia-inducible factor $1 \alpha$ (HIF-1 $\alpha$ ) expression in myocardial cells (A and B) Brown color indicates HIF-1 $\alpha$ staining and blue color indicates myocardial nuclear staining. Magnification (A) x200 and (B) x400. (C) Western blot analysis revealed that the level of HIF-1 $\alpha$ expression was markedly elevated in the AAV-PR39-treated group. (D) The mean optical density (OD) value of HIF-1 $\alpha$ in the AAV-PR39-treated group was markedly higher than that in the control (PBS-treated) group. ${ }^{* *} \mathrm{P}<0.01$ compared with the control group.

and FGF (9). In this study, we found that PR39 regulated the HIF-1 $\alpha$-induced expression of angiogenic growth factors. The observed profile of gene expression is an accordance with the observed pro-angiogenic effect, such as increased regional blood flow. It has been reported that PR39 augments the expression of HIF-1 $\alpha$-dependent genes, such as VEGF and its receptor, VEGF-R1, as well as that of HIF-1 $\alpha$-independent genes, such as VEGF-R2, FGFR-1 and syndecan-4 (8). These results suggested that PR39 regulated the HIF- $1 \alpha$-induced expression of angiogenic growth factors.

We further demonstrated that the apoptotic rate was significantly lower in the AAV-PR39-treated group, suggesting that PR39 inhibited apoptosis. A previous study reported that exogenous PR39 inhibits apoptosis in endothelial cells subjected to hypoxic injury, and that the anti-apoptotic effects of PR39 are mediated by the increased inhibitor of apoptosis protein (IAP)-2 expression via transcriptional and post-transcriptional regulation (12). The establishment of an effective collateral circulation (through the induction of anti-apoptotic effects) during the early stages of myocardial infarction is conducive for the reduction of the infarct size and the protection of heart functions.

Previous studies have demonstrated that both apoptosis and necrosis exist in myocardial infarction. During the early stages 
of myocardial infarction, apoptosis is the main type of cell death observed $(17,19)$. Thus, during the early stages of acute myocardial infarction, the disruption of the apoptotic signal transduction pathways appears to be an effective approach to reducing cell death in the ischemic area and to protect heart functions $(13,19)$. Damage to the myocardial cells surrounding the myocardial infarct area is reversible and part of the myocardial tissues maintains the systolic and diastolic function (20). At this point, timely blood supply can recover the function of the myocardial tissues. However, if the ischemic injury is continuously increased, myocardial damage becomes irreversible (21). Therefore, prompt treatment can limit the myocardial infarct size and reduce mortality in patients with myocardial infarction. Reversing the damage to myocardial cells is dependent on the supply of oxygen and the removal of metabolites. Rapid establishment of collateral circulation in the ischemic region can markedly improve the blood supply and reduce the myocardial infarct size.

During the early stages of myocardial infarction, PR39 can rescue the cells in the ischemic region through its antihypoxic and anti-apoptotic functions (22). It also enhances the expression of myocardial HIF-1 $\alpha$, which further promotes local angiogenesis and accelerates the establishment of collateral circulation in the ischemic areas. Therefore, PR39 has a wide range of clinical applications in the early treatment of the ischemic myocardium and can be used to prevent myocardial infarction. In this study, we demonstrated that the left ventricular myocardial infarct area in the pigs in the AAV-PR39-treated group was significantly reduced, further demonstrating the safety and efficacy of PR39 in the treatment of ischemic heart disease.

In conclusion, the expression of recombinant PR39 elevates the levels of HIF-1 $\alpha$ under hypoxic conditions and decreases the infarct size by upregulating the expression of HIF-1 $\alpha$ in the surrounding areas of the infarct. As a masterswitch of angiogenesis, PR39 can effectively initiate angiogenic mechanisms, rapidly establish collateral circulation, protect the viability of myocardial cells and reduce the infarct size. The results obtained in this study provide new insight into the genetic therapy of coronary heart disease.

\section{Acknowledgements}

The authors thank Mr. Guangxiao Yang and Ms. Quanying Wang (Xi'an Huaguang Bioengineering Corp., Xi'an, Shaanxi, China) for assisting with the experiments.

\section{References}

1. Thygesen K, Alpert JS, Jaffe AS, Simoons ML, Chaitman BR and White HD: Third universal definition of myocardial infarction. J Am Coll Cardiol 60: 1581-1598, 2012.

2. Kanashiro-Takeuchi RM, Schulman IH and Hare JM: Pharmacologic and genetic strategies to enhance cell therapy for cardiac regeneration. J Mol Cell Cardiol 51: 619-625, 2011.

3. Carmeliet P and Jain RK: Molecular mechanisms and clinical applications of angiogenesis. Nature 473: 298-307, 2011.

4. Potente M, Gerhardt H and Carmeliet P: Basic and therapeutic aspects of angiogenesis. Cell 146: 873-887, 2011
5. Lykouras D, Flordellis C and Dougenis D: Gene therapy targets and the role of pharmacogenomics in heart failure. In: Targets in Gene Therapy. You Y (ed.). InTech. doi: 10.5772/20004. 2011. Available at: http://www.intechopen.com/ books/targets-in-gene-therapy/gene-therapy-targets-and-the-roleof-pharmacogenomics-in-heart-failure.

6. Lee RJ, Springer ML, Blanco-Bose WE, Shaw R, Ursell PC and Blau HM: VEGF gene delivery to myocardium: deleterious effects of unregulated expression. Circulation 102: 898-901, 2000.

7. Muinck E, Nagy N, Tirziu D, Murakami M, Gurusamy N, Goswami SK, Ghatpande S, Engelman RM, Simons M and Das DK: Protection against myocardial ischemia-reperfusion injury by the angiogenic Masterswitch protein PR 39 gene therapy: the roles of HIF1alpha stabilization and FGFR1 signaling. Antioxid Redox Signal 9: 437-445, 2007.

8. Li J, Post M, Volk R, Gao Y, Li M, Metais C, Sato K, Tsai J, Aird W and Rosenberg RD: PR39, a peptide regulator of angiogenesis. Nat Med 6: 49-55, 2000.

9. Cavadas MA, Nguyen LK and Cheong A: Hypoxia-inducible factor (HIF) network: insights from mathematical models. Cell Commun Signal 11: 42-56, 2013.

10. Lee PH, Ohtake T, Zaiou M, Murakami M, Rudisill JA, Lin KH and Gallo RL: Expression of an additional cathelicidin antimicrobial peptide protects against bacterial skin infection. Proc Natl Acad Sci USA 102: 3750-3755, 2005

11. Shen F, Fan Y, Su H, Zhu Y, Chen Y, Liu W, Young WL and Yang GY: Adeno-associated viral vector-mediated hypoxiaregulated VEGF gene transfer promotes angiogenesis following focal cerebral ischemia in mice. Gene Ther 15: 30-39, 2007.

12. Bao J, Sato K, Li M, Gao Y, Abid R, Aird W, Simons M and Post MJ: PR-39 and PR-11 peptides inhibit ischemia-reperfusion injury by blocking proteasome-mediated $\mathrm{I} \kappa \mathrm{B} \alpha$ degradation. Am J Physiol Heart Circ Physiol 281: H2612-H2618, 2001.

13. Korthuis RJ, Gute DC, Blecha F and Ross CR: PR-39, a proline/ arginine-rich antimicrobial peptide, prevents postischemic microvascular dysfunction. Am J Physiol 277: H1007-H1013, 1999.

14. Li Y, Qiu S, Song L, Yan Q and Yang G: Secretory expression of p53 (N15)-Ant following lentivirus-mediated gene transfer induces cell death in human cancer cells. Cancer Invest 26: 28-34, 2008.

15. Garrett DJ, Cohen JC and Larson JE: Long term physiologic modification using rAAV in utero gene-therapy. Genet Vaccines Ther 2: 4, 2004.

16. Zaiss AK, Liu Q, Bowen GP, Wong NC, Bartlett JS and Muruve DA: Differential activation of innate immune responses by adenovirus and adeno-associated virus vectors. J Virol 76: 4580-4590, 2002.

17. Carmeliet P, Dor Y, Herbert JM, Fukumura D, Brusselmans K, Dewerchin M, Neeman M, Bono F, Abramovitch R, Maxwell P, Koch CJ, Ratcliffe P, Moons L, Jain RK, Collen D and Keshert E: Role of HIF-1alpha in hypoxia-mediated apoptosis, cell proliferation and tumour angiogenesis. Nature 394: 485-490, 1998.

18. Gerber HP, Condorelli F, Park J and Ferrara N: Differential transcriptional regulation of the two vascular endothelial growth factor receptor genes Flt-1, but not Flk-1/KDR, is up-regulated by hypoxia. J Biol Chem 272: 23659-23667, 1997.

19. Wu J, Parungo C, Wu G, Kang PM, Laham RJ, Sellke FW, Simons M and Li J: PR39 inhibits apoptosis in hypoxic endothelial cells. Circulation 109: 1660-1667, 2004.

20. Brevoord D, Kranke P, Kuijpers M, Weber N, Hollmann M and Preckel B: Remote ischemic conditioning to protect against ischemia-reperfusion injury: a systematic review and meta-analysis. PLoS One 7: e42179, 2012.

21. Masci PG, Francone M, Desmet W, Ganame J, Todiere G, Donato R, Siciliano V, Carbone I, Mangia M and Strata E: Right ventricular ischemic injury in patients with acute ST-segment elevation myocardial infarction clinical perspective characterization with cardiovascular magnetic resonance. Circulation 122: 1405-1412, 2010

22. Post MJ, Sato K, Murakami M, Bao J, Tirziu D, Pearlman JD and Simons M: Adenoviral PR39 improves blood flow and myocardial function in a pig model of chronic myocardial ischemia by enhancing collateral formation. Am J Physiol Regul Integr Comp Physiol 290: R494-R500, 2006. 